\title{
Desempenho produtivo da tilápia do nilo em tanques-rede numa represa pública: modelo empírico de classificação
}

\author{
Productive development of Nile tilapia raised in net cages at a public reservoir: \\ brief analysis of the empirical model of classification
}

\section{Edmir Daniel Carvalho ${ }^{\mathrm{I}}$ André Luiz Scarano Camargo ${ }^{\mathrm{II}}$ Augusto Seawright Zanatta ${ }^{\mathrm{III}}$}

\section{RESUMO}

Nos últimos anos, a piscicultura brasileira em tanques-rede, em águas públicas abertas, tem sido alavancada por incentivos governamentais e privados, e a tilápia do nilo (Oreochromis niloticus) tem sido o principal pescado utilizado. Como forma de melhorar a eficiência de produção, empiricamente, os piscicultores realizam o manejo de classificação e a repicagem das tilápias em engorda, resultando em lotes com diferentes rendimentos $e$ precocidades, num gradiente dos melhores aos piores rendimentos. Este trabalho objetivou avaliar o desempenho de engorda de tilápias em um rotineiro sistema de produção em tanques-rede, sendo utilizado o usual modelo empírico de classificação e repicagem em três etapas distintas e tendo por fundamento a separação dos lotes de peixes em classes de tamanho e peso, ao longo do processo de engorda. Os resultados mostraram situações bem distintas. Num extremo, lotes de peixes precoces sendo comercializados após o processo de classificação e repicagem, aos 152 dias de engorda, apresentando taxas crescentes de ganho de peso diário (entre 0,30 e $7,23 \mathrm{~g}$ dia $^{-1}$ ) e de conversão alimentar aparente satisfatória (entre 1,50 e 1,53). No outro extremo, lotes heterogêneos sendo comercializados com 213 dias, taxas de ganho de peso inconstantes (entre 0,94 e 8,24) e elevadas taxas de conversão alimentar aparente (entre 1,54 e 3,03). A partir dos resultados, pode-se destacar a relevância dessa prática empírica de manejo zootécnico em piscicultura, que mostra grandes discrepâncias de rendimentos e precocidades entre os lotes, considerando o tempo de cultivo, o ganho de peso e a conversão alimentar aparente. Além disso, recomenda-se, para os lotes heterogêneos destinos, alternativas como a utilização de piscicultura extensiva (sem arraçoamento) para a mitigação dos impactos ambientais negativos inerentes dessa atividade zootécnica.

Palavras-chave: Oreochromis niloticus, tilapicultura,
desempenho produtivo, impactos ambientais, repicagem.

\section{ABSTRACT}

In the past few years Brazilian aquaculture in net cages located at public reservoirs has been increasing due to industry and government efforts. Nile tilapia (Oreochromis niloticus) has been the main fish species used in this system. Aiming to increase production efficiency, fish farmers empirically apply classification and grading management of finishing tilapias resulting in groups with different performances and precocity, in a grade basis from the best to worst performances. This study aimed to evaluate growth performance of finishing tilapias raised in a common net cage production system using the usual empirical model of classification and grading in three distinct steps along the finish phase. Results showed two distinct situations: the first with rapid growth groups of fish being marketed at 152 days presenting increasing daily weight gain (between 0.30 and $7.23 \mathrm{~g} \mathrm{day}^{-1}$ ) and proper feed conversion ratios (between 1.50 and 1.53); and the second with fish being marketed at 213 days presenting not constant weight gains (between 0.94 and 8.24) and high feed conversion ratios (between 1.54 and 3.03). Based on the results it must be emphasizing the importance of this empirical fish management due to the high variability in

IDepartamento de Morfologia, Universidade Estadual Paulista (UNESP), 18618-000, Botucatu, SP, Brasil. E-mail: carvalho@ibb.unesp.br. Autor para correspondência.

"Programa de Pós-graduação em Aquicultura, do Centro de Aquicultura, UNESP (CAUNESP), Jaboticabal, SP, Brasil.

IIIPrograma de Pós-graduação em Ciências Biológicas, Zoologia, Laboratório de Biologia e Ecologia de Peixes, Departamento de Morfologia, Instituto de Biociências, UNESP, Botucatu, SP, Brasil. 
the growth performance among fish groups. Nevertheless, the appropriate strategy recommended for the use of heterogeneous groups of fish is the extensive fish farming system, which aims to mitigate the environmental impact caused by the excessive feeding used as the purpose for a high production.

Key words: Oreochromis niloticus, tilapiculture, productive performance, environmental impacts, grading.

\section{INTRODUÇÃO}

Nos dias atuais, a aquicultura vem tornando-se importante fonte de alimento de origem animal, devido à intensa pressão nos estoques pesqueiros naturais. KUBITZA (2007) discute que a exploração dos recursos pesqueiros, em escala mundial, passou por um decréscimo de mais de $30 \%$ (entre 1975 a 2005), enquanto que a aquicultura torna-se responsável por suprir a demanda de alimento. Sabe-se que essa atividade, quando bem planejada, é benéfica para o desenvolvimento econômico do país; entretanto, há necessidade do aprimoramento das técnicas de cultivo e também do seu ordenamento (AGOSTINHO et al., 2007), embasando-se no trinômio: 1) produção, 2) conservação dos recursos naturais e 3) desenvolvimento social (VALENTI, 2000). Dados da FAO (2006) destacam que a taxa de crescimento por ano da aquicultura supera todas as outras atividades zootécnicas, em que o Brasil apresenta crescimento superior a $25 \%$ ao ano.

Apenas em 2006 mais de um milhão de toneladas de pescado foram produzidas no Brasil, e a aquicultura continental contribuiu com 18,2\% desse montante (CARVALHO-FILHO, 2008), mesmo sendo utilizadas técnicas ainda rudimentares. Essa complexa atividade exige aprendizados constantes, assistência técnica especializada e contínua e, principalmente, suporte científico (AGOSTINHO et al., 2007).

O principal peixe cultivado em tanquesrede, em escala mundial, é a tilápia (Oreochromis niloticus, Trewavas, 1983) (FAO, 2006), em razão de suas excelentes características organolépticas, da tolerância a diferenças ambientais, da alta taxa de conversão alimentar e da adaptação à criação praticada em elevada densidade (AYROZA et al., 2008).

A viabilidade econômica dessa atividade zootécnica está associada, entre outros aspectos, ao manejo e gerenciamento, não bastando ter apenas bons viveiros a baixo custo, sendo preciso observar também a quantidade e qualidade da água, o manejo da densidade e o tipo de ração (MARTIN et al., 1995). Dessa forma, a densidade de estocagem destaca-se como uma importante variável que tem reflexos nas práticas de manejo e na rentabilidade comercial do processo, portanto, estudada por vários autores (CARNEIRO et al., 1999; CAVERO et al., 2003). Esses autores salientam que densidades de estocagem ideal podem quebrar a heterogeneidade, em termos de tamanho e peso dos lotes de peixes em engorda, influenciando a homogeneidade do pescado final. Dessa forma, as criações em tanques-rede de pequeno volume, quando em densidades adequadas, podem ser zootecnicamente vantajosas pelo fato de suportarem altas densidades de estocagem.

Entretanto, essa informação é vaga, indicando um viés, tanto que muitos piscicultores em sistema de tilapicultura em tanques-rede, no Brasil, vêm aplicando nova técnica de manejo, empiricamente fundamentada no crescimento heterogêneo e na hierarquia de dominância que os ciclídeos apresentam (VOLPATO \& FERNANDES, 1994). Também é pertinente ressaltar que esta e outras situações mostram que a atividade de piscicultura em tanques-rede necessita ainda de muito conhecimento científico e apoio técnico basal (AGOSTINHO et al., 2007), uma vez que grande parte desses empreendimentos são fadados ao fracasso, tornando-se inviável economicamente em curto e médio prazo. Podem ser destacados dois fatores imprescindíveis ao não sucesso do empreendimento: $1^{\circ}$ ) falta de mão-de-obra qualificada (técnicos em aquicultura e correlatos) e $2^{\circ}$ ) conforme já preconizado por BEVERIDGE (1996), boas práticas de manejo, que são retroalimentadas pelo $1^{\circ}$ fator.

No intuito de aprimorar as técnicas de manejo que visam a garantir a viabilidade e o sucesso dessa atividade zootécnica, os objetivos deste trabalho foram descrever e avaliar o desempenho de engorda de tilápias em um sistema de produção comercial em tanques-rede, sendo utilizados o modelo empírico de classificação e repicagem por classes de tamanho dos indivíduos.

\section{MATERIAL E MÉTODOS}

A coleta de dados foi realizada em um empreendimento particular, no Município de Buritama, São Paulo (SP), tendo cerca de 80 tanquesrede numa área de dois hectares, localizada nas margens do reservatório da UHE de Nova Avanhandava (baixo Rio Tietê). Os alevinos de 
tilápias (Oreochromis niloticus) eram provenientes de uma unidade produtora da própria empresa. A coleta de dados foi realizada em três etapas empregadas normalmente no manejo e tem por fundamento separar os lotes de peixes em classes de tamanho ao longo da engorda:

Fase 01 - Em julho/2004, foram estocados 12.000 juvenis com grande heterogeneidade de tamanho, de linhagem monosexuada masculina, com peso estimado de $24,0 \mathrm{~g}$ em dois tanques-rede (A e B - com $6 \mathrm{~m}^{3}$ ), resultando na densidade de 500 peixes $\mathrm{m}^{-3}$ tanque $^{-1}$. Essa etapa durou 93 dias e se caracterizou pela maior diferença de tamanho entre os juvenis.

Fase 02 - Aos 93 dias de experimento, quando os maiores atingiram peso de $120,0 \mathrm{~g}$, os lotes de peixes dos tanques A e B foram separados e classificados em dois grupos distintos (grandes e médios/pequenos), e o lote do tanque A foi subdividido para dois outros tanques: A1 (peixes de maior porte) e A2 (peixes restantes = tamanhos ainda heterogêneos). O mesmo procedimento foi executado para o lote do tanque $\mathrm{B}$.
Os lotes dos tanques A1 e B1 não sofreram nova classificação, permanecendo nesses tanques até a despesca e comercialização, aos 153 dias. Ainda, os lotes heterogêneos (A2 e B2) permaneceram por 44 dias nessa fase.

Fase 03 - Aos 137 dias, os lotes A2 e B2 foram submetidos a novo processo de classificação e repicagem. Assim, os peixes do tanque A2 foram distribuídos para dois outros tanques, e no tanque A3 foram estocados os peixes medianos, e os peixes menores foram estocados no tanque A4. O mesmo procedimento também foi executado para os peixes do tanque B2. A figura 1 esquematiza esses procedimentos.

A partir desse último manejo classificatório, esses quatro lotes (A3 e A4; B3 e B4) mantiveram-se estocados nesses tanques até o momento de sua despesca e comercialização. Manejo geral - Os peixes foram alimentados com ração artificial balanceada extrusada da marca FriAcqua, com taxas de arraçoamento variando de 2 a $6 \%$ do peso vivo (BARBOSA et al., 2005). Nas fases 01 e 02 , os peixes foram alimentados quatro vezes

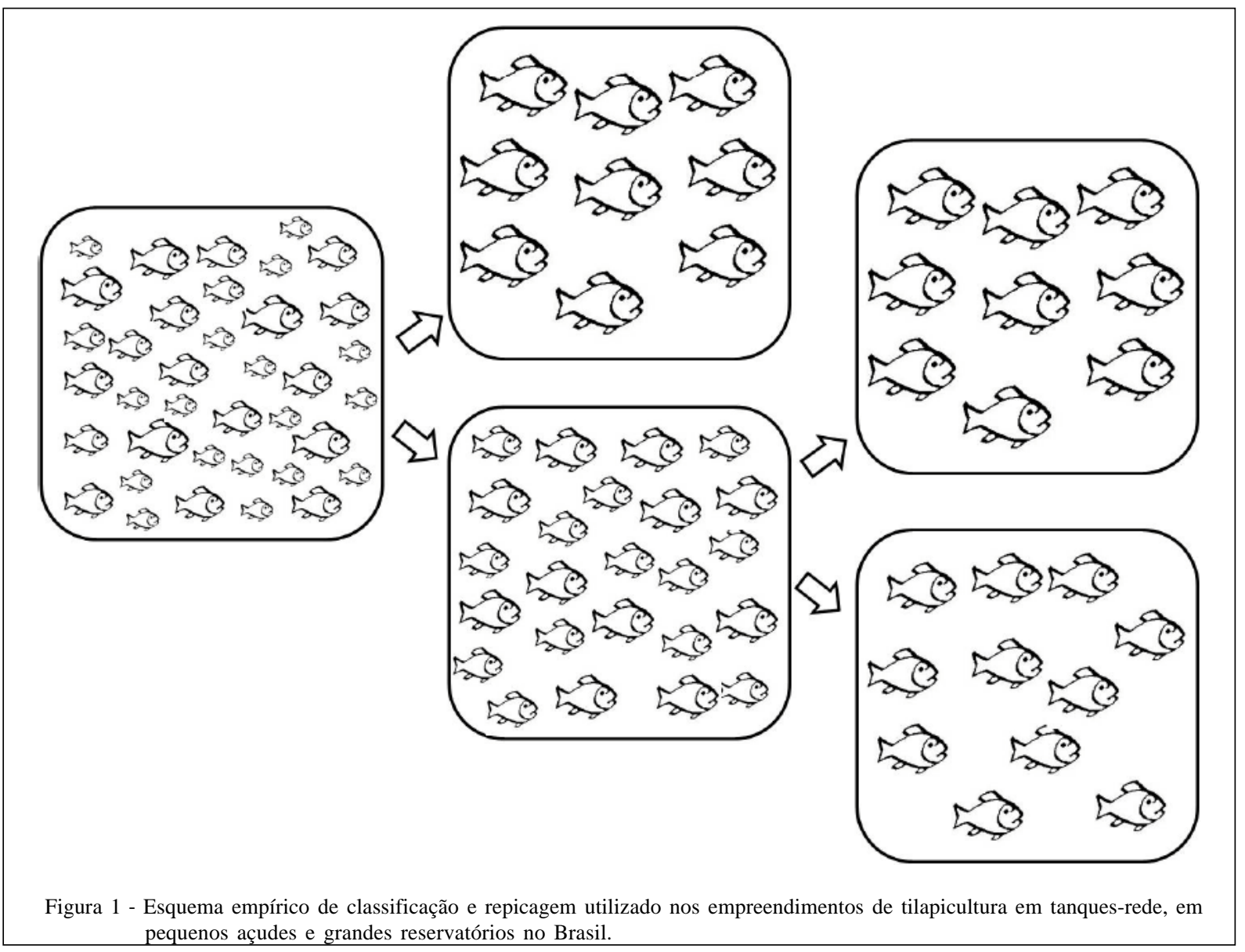

Ciência Rural, v.40, n.7, jul, 2010. 
ao dia com ração contendo 36,0\% PB (proteína bruta) e, na fase 3 , duas vezes ao dia com ração contendo $32,0 \%$ PB.

Análise do desempenho zootécnico Foram realizadas amostragens, ao acaso, a cada 30 dias. Para tanto, foram coletados 80 indivíduos por lote para avaliação biométrica (comprimento padrão - Ls (cm) e peso total - Wt (gramas)). Depois desse procedimento, os exemplares foram devolvidos aos seus tanques. Com base em CARVALHO (1992), foram determinados o peso médio total (Wt), o ganho de peso diário (GPD), a densidade de estocagem (DE), a taxa de conversão alimentar (TCA), e a taxa de sobrevivência (TS).

\section{RESULTADOS E DISCUSSÃO}

No presente estudo, o processo de engorda teve início com $\mathrm{DE}$ de 500 peixes $\mathrm{m}^{-3}$ tanque ${ }^{1}$, em que, na primeira fase do experimento (T1 e T2), o GPD dos lotes variou entre 0,30 e 1,99g dia $^{-1} \mathrm{e}$, nas fases seguintes, após o processo de classificação e repicagem reduzindo a $\mathrm{DE}$, os valores de GPD aumentaram (Tabelas 1 e 2). Assim, após a primeira repicagem, aos 93 dias de engorda, o GPD aumentou, variando entre 0,94 e 7,23g dia ${ }^{-1}$ em T4. Além disso, após o segundo processo de repicagem e redução populacional, o GPD aumentou ainda mais (entre 2,13 e 8,24g dia-1 , a partir de T5).
Quando são comparados esses valores de GPD globais com dados disponíveis na literatura, constata-se que as taxas mensuradas no presente estudo foram mais elevadas. Por exemplo, CARNEIRO et al. (1999), em estudo similar com tilápia vermelha em tanques-rede, na região de Jaboticabal (SP), mensuraram GPD menores (entre 0,90 a $\left.0,94 \mathrm{~g} \mathrm{dia}^{-1}\right)$. Contudo, em condições climáticas distintas e favoráveis à tilapicultura em tanquesrede (região Nordeste do Brasil), autores como SAMPAIO et al. (2005) e MORAES et al. (2006) determinaram boas taxas de GPD (4,73 a 5,02g dia ${ }^{-1} \mathrm{e}$ 5,20 a $5,67 \mathrm{~g} \mathrm{dia}^{-1}$, respectivamente), porém ainda menores que as taxas deste trabalho, no qual foi realizado o processo de classificação e repicagem.

De acordo com CONTE (2002), para a piscicultura, o gasto com a compra de ração comercial balanceada pode chegar a $70 \%$ do custeio dessa atividade. Dessa forma, é de extrema importância buscar meios de minimizar esses custos, por meio de diagnósticos precisos das taxas de conversão alimentar aparente (TCA) e do uso de técnicas de manejo que visem à redução são imprescindíveis.

Para os lotes dos tanques e das rotas A A1 e B - B1, submetidos à apenas uma classificação e repicagem, foram estimadas, para o momento da despesca, taxas de conversão alimentar aparente (TCA) de 1,50 e 1,53, respectivamente (Tabelas 1 e 2 ).

Tabela 1 - Avaliações zootécnicas do cultivo de Oreochromis niloticus em tanques-rede, no processo de classificação e repicagem.

\begin{tabular}{|c|c|c|c|c|c|c|c|}
\hline \multirow[b]{2}{*}{ Variáveis } & \multirow[b]{2}{*}{ Rotas } & \multicolumn{6}{|c|}{ 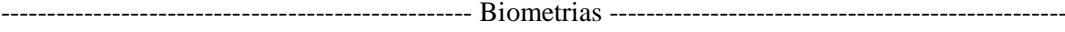 } \\
\hline & & T0 & $\mathrm{T} 1$ & $\mathrm{~T} 2$ & $\mathrm{~T} 3 ?$ & $\mathrm{~T} 4 ?$ & $\mathrm{~T} 5 ?$ \\
\hline \multirow{3}{*}{$\mathrm{DE}\left(\mathrm{n} \mathrm{m}^{-3}\right)$} & I & 500 & 482 & 480 & 472 & 154 & 152 \\
\hline & II & 500 & 482 & 480 & 472 & 311 & 142 \\
\hline & III & 500 & 482 & 480 & 472 & 311 & 145 \\
\hline \multirow{3}{*}{ Wt médio (g) } & I & 24,00 & 49,64 & 58,62 & 130,21 & 334,93 & 516,95 \\
\hline & II & 24,00 & 49,64 & 58,62 & 130,21 & 158,35 & 390,66 \\
\hline & III & 24,00 & 49,64 & 58,62 & 130,21 & 158,35 & 236,79 \\
\hline \multirow{3}{*}{ GPD $\left(\mathrm{g} \mathrm{d}^{-1}\right)$} & I & - & 0,85 & 0,30 & 2,39 & 6,82 & 6,07 \\
\hline & II & - & 0,85 & 0,30 & 2,39 & 0,94 & 7,74 \\
\hline & III & - & 0,85 & 0,30 & 2,39 & 0,94 & 2,61 \\
\hline \multirow{3}{*}{ TCA } & I & - & - & - & - & - & 1,50 \\
\hline & II & - & - & - & - & - & 2,70 \\
\hline & III & - & - & - & - & - & 1,54 \\
\hline \multirow{3}{*}{ TS (\%) } & I & - & - & - & - & - & 91 \\
\hline & II & - & - & - & - & - & 88 \\
\hline & III & - & - & - & - & - & 90 \\
\hline
\end{tabular}

† Classificação e repicagem, sendo a $1^{\underline{a}}$ entre T3 e T4 e a $2^{\underline{a}}$ entre T4 e T5; Despesca e comercialização aos 152 dias de engorda; $\Delta$ T $\cong 30$ dias. I = Lote dos tanques A-A1; II=Lote dos tanques A-A2-A3; III=Lote dos tanques A-A2-A4.

Ciência Rural, v.40, n.7, jul, 2010. 
Tabela 2 - Avaliações zootécnicas do cultivo de Oreochromis niloticus em tanques-rede, no processo de classificação e repicagem.

\begin{tabular}{|c|c|c|c|c|c|c|c|c|c|}
\hline \multirow{2}{*}{ Variáveis } & \multirow{2}{*}{ Rotas0 } & \multirow[b]{2}{*}{ T0 } & \multirow[b]{2}{*}{$\mathrm{T} 1$} & \multirow[b]{2}{*}{$\mathrm{T} 2$} & \multirow[b]{2}{*}{ T3? } & \multirow[b]{2}{*}{$\mathrm{T} 4 ?$} & \multirow[b]{2}{*}{$\mathrm{T} 5 ?$} & \multirow[b]{2}{*}{ T6 } & \multirow[b]{2}{*}{$\mathrm{T} 7$} \\
\hline & & & & & & & & & \\
\hline \multirow{4}{*}{$\begin{array}{l}\mathrm{DE} \\
\left(\mathrm{n} \mathrm{m}^{-3}\right)\end{array}$} & $\mathrm{IV}^{*}$ & 500 & 480 & 479 & 471 & 155 & 151 & - & - \\
\hline & $\mathrm{V}^{* *}$ & 500 & 480 & 479 & 471 & 321 & 145 & 145 & 145 \\
\hline & $\mathrm{VI}^{* * *}$ & 500 & 480 & 479 & 471 & 312 & 145 & - & - \\
\hline & $\mathrm{IV}^{*}$ & 24,00 & 47,81 & 80,61 & 109,04 & 325,94 & 519,47 & - & - \\
\hline \multirow{3}{*}{$\begin{array}{l}\text { Wt } \\
\text { médio (g) }\end{array}$} & $\mathrm{V}^{* *}$ & 24,00 & 47,81 & 80,61 & 109,04 & 170,00 & 376,91 & 545,67 & 615,34 \\
\hline & $\mathrm{VI} * * *$ & 24,00 & 47,81 & 80,61 & 109,04 & 170,00 & 233,79 & 480,94 & - \\
\hline & $\mathrm{IV}^{*}$ & - & 0,79 & 1,09 & 0,95 & 7,23 & 6,45 & - & - \\
\hline \multirow{3}{*}{$\begin{array}{l}\text { GPD } \\
\left(\mathrm{g} \mathrm{d}^{-1}\right)\end{array}$} & $\mathrm{V}^{* *}$ & - & 0,79 & 1,09 & 0,95 & 2,03 & 6,90 & 5,63 & 2,32 \\
\hline & $\mathrm{VI}^{* * *}$ & - & 0,79 & 1,09 & 0,95 & 2,03 & 2,13 & 8,24 & - \\
\hline & $\mathrm{IV}^{*}$ & - & - & - & - & - & - & - & 1,53 \\
\hline \multirow[t]{3}{*}{ TCA } & $\mathrm{V}^{* *}$ & - & - & - & - & - & - & - & 3,03 \\
\hline & VI*** & - & - & - & - & - & - & - & 1,54 \\
\hline & $\mathrm{IV}^{*}$ & - & - & - & - & - & - & - & 93 \\
\hline \multirow[t]{2}{*}{ TS (\%) } & $\mathrm{V}^{* *}$ & - & - & - & - & - & - & - & 91 \\
\hline & $\mathrm{VI} * * *$ & - & - & - & - & - & - & - & 93 \\
\hline
\end{tabular}

† Classificação e repicagem, sendo a $1^{\text {a }}$ entre T3 e T4 e a $2^{-a}$ entre T4 e T5; *Despesca e comercialização aos 152 dias de engorda; **Despesca e comercialização aos 213 dias de engorda; ***Despesca e comercialização aos 183 dias de engorda $\Delta$ T $\cong 30$ dias; IV=Lote dos tranques B-B1; V=Lotes dos tanques B-B2-B3; VI=Lotes dos tanques B-B2-B4.

Novamente, contrapondo esses resultados aos de MARENGONI (2006), constata-se que, nas rotas do presente trabalho, as TCAs são ligeiramente melhores, considerando que o referido autor estimou TCAs entre 1,54 a 1,75.

Entretanto, quando são comparados os resultados de CARNEIRO et al. (1999) e de BOZANO et al. (1999), a vantagem do processo de classificação e repicagem fica bastante evidente. Isso porque os valores de TCA obtidos por CARNEIRO et al. (1999) foram extremamente elevados (entre 3,04 à 3,29), correspondendo a taxas determinadas para o lote/a rota dos tanques B-B2-B3 (TCA=3,03), que confinava os peixes de menor porte e maior tempo de engorda (183 dias). Ainda, BOZANO et al. (1999), avaliando o desempenho zootécnico de tilápias nilóticas em tanques-rede, estimaram TCA superiores (entre 2,96 à 6,81).

Pode-se inferir que o processo tradicional de cultivo (sem classificação e repicagem) de tilápia, independente da linhagem genética, da qualidade das rações e das condições ambientais, esteja diretamente relacionado com o crescimento heterogêneo e a hierarquia de dominância (VOLPATO \& FERNANDES, 1994), o que reflete em altas taxas de conversão alimentar aparente. Somando-se a essa hipótese, o manejo zootécnico, principalmente o alimentar incorreto, em que há grandes desperdícios de ração, também pode contribuir para essa situação. Além disso, a transferência do processo tradicional de cultivo ao piscicultor já é um indicativo de sua inviabilidade comercial. Para sanar esse último problema, MEURER et al. (2003) recomenda a execução de manejo alimentar rigoroso, com base em biometrias sequenciais e contínuas, para o ajuste preciso das taxas de arraçoamento. Portanto, no contexto da viabilidade desse processo de produção animal, estas e outras técnicas de manejo são imprescindíveis ao piscicultor.

Em relação às taxas de sobrevivência (TS), ABOU et al. (2007) reportam que a TS não é dependente da DE, em contraposição, já YI et al. (1996) consideram que a baixa TS diagnosticada $(57,2 \%)$ em seus estudos estaria correlacionada diretamente com as altas DE, que, por sua vez, promoveriam alto consumo de oxigênio dissolvido no período noturno, com reflexos nas primeiras horas da manhã. Neste estudo, obteve-se uma TS satisfatória, pois variou de 88 a 93\% (Tabelas 1 e 2). MARENGONI (2006), avaliando o desempenho zootécnico de tilápias em DE similares ao presente estudo, quantificou valores de TS ligeiramente superiores (98,3 a 99,1\%), ao passo que ABOU et al. (2007) determinaram valores de TS bem inferiores (74,0 a 78,9\%), mas afirmam que tais valores não foram decorrentes da DE. 
É de senso comum que atualmente o peso médio do pescado (tilápias) procurado pelo mercado consumidor encontra-se em torno de $500 \mathrm{~g} / 600 \mathrm{~g}$ até $1 \mathrm{~kg}$, dependendo do mercado consumidor (RIBEIRO, 2007). Nesse contexto, os lotes com pescado de maior porte (lotes dos tanques A-A1 e B-B1) atingiram rapidamente (aos 123 dias de cultivo) esses valores médios (de 516,9 e 519,5g, respectivamente). Ao contrário, os lotes mais heterogêneos apresentavam quase a metade dos valores de peso $(233,8$ a 390,7g) no mesmo período de cultivo (Tabelas 1 e 2).

Nesse estado atual, em que a aquicultura brasileira ainda espera o salto quali-quantitativo, são apresentadas as seguintes reflexões e ações: é valido executar todo o processo de engorda com os lotes heterogêneos que apresentaram altas taxas de conversão alimentar aparente? Ou seria conveniente dar outros destinos economicamente e/ou ambientalmente viáveis? Por exemplo, aplicação de técnicas de biomanipulação (STARLING \& LAZZARO, 2001) desses lotes heterogêneos de baixo rendimento zootécnico, sendo utilizada: a piscicultura extensiva (sem arraçoamento), cujos efeitos serão traduzidos na mitigação dos impactos ambientais negativos inerentes dessa atividade zootécnica. Ou ainda, analogamente poder-se-ia proceder ao retorno desse pescado de alto custo (tilápias nanicas) como insumos na fabricação de rações, com base nos estudos de GYLLENHAMMAR et al. (2008), os quais demonstraram que o uso de $11 \%$ da biomassa de peixes nativos agregados aos sistemas de tanquesredes marinhos pode zerar as emissões de fósforo orgânico ao meio aquático.

Para concluir, a tese de AGOSTINHO et al. (2007) é incontestável no enfoque de que os maiores desafios para o aprimoramento da aquicultura nacional a longo prazo, em grandes reservatórios, resume-se na busca de soluções paralelas aos seguintes entraves: o uso de espécies nativas como modelo zootécnico viável; o controle efetivo dos escapes das exóticas ainda em uso, e a mitigação das emissões de efluentes advindos dessa atividade. Para tanto, o pano de fundo dessa conflituosa questão seria "como combinar nossas diferenças com a unidade de determinada vida coletiva” (Touraine, 1998, apud FREITAS \& ANNUNCIATO, 2008).

\section{AGRADECIMENTOS}

Agradecemos às seguintes instituições e empresas: à FAPESP (proc. $\mathrm{n}^{\circ}$ 03/11239-2), pelo suporte financeiro); ao CNPq, pela bolsa de produtividade em pesquisa ao primeiro autor; ao CT-hidro CNPq, pela bolsa do terceiro autor; ao empreendimento Escama Forte Piscicultura Ltda. (Município de Buritama, SP), que cedeu suas instalações e forneceu o apoio técnico e o material desta pesquisa.

\section{REFERÊNCIAS}

ABOU, Y. et al. Effects of stocking density on growth, yield and profitability of farming Nile tilapia, Oreochromis niloticus L, fed Azolla diet, in earthen ponds. Aquaculture Research, Oxford, v.38, p.595604, 2007.

AgOstinho, A.A. et al. Ecologia e manejo de recursos pesqueiros em reservatórios do Brasil. Maringá: Eduem, 2007. 502p.

AYROZA, L.M.S. et al. Piscicultura no médio Paranapanema: situação e perspectivas. Assis, 2008. Acesso em: 20 out. 2008. Online. Disponível em: http:// www.pesca.sp.gov.br/list_arquivos.php?pag=1.

BARBOSA, A.C.A. et al. Avaliação de diferentes seqüências de arraçoamento no desenvolvimento de tilápias cultivadas em gaiolas. Natal: Empresa de Pesquisa Agropecuária do Rio Grande do Norte, 2005. 14p. (Boletim de Pesquisa e desenvolvimento, 31).

BEVERIDGE, M.C.M. Cage aquaculture. London: Fishing News Books, 1996. 346p.

BOZANO, G.L.N. et al. Desempenho da tilápia nilótica Oreochromis niloticus (L.) em gaiolas de pequeno volume. Scientia Agricola, Piracicaba, v.56, n.4, p.819825, 1999. Disponível em: <http://www.scielo.br/ scielo.php? script=sci_arttext\&pid=S 0103 $90161999000400008 \& \operatorname{lng}=\mathrm{en} \& \mathrm{nrm}=\mathrm{iso}>$. Acesso em: 25 maio, 2010. doi: 10.1590/S010390161999000400008 .

CARNEIRO, P.C.F. et al. Produção da tilápia vermelha da Flórida em tanques-rede. Scientia Agricola, Piracicaba, v.56, n.3, p.673-679, 1999. Disponível em: <http:// www.scielo.br/scielo.php?script=sci_arttext\&pid=S010390161999000300023\&lng $=$ en $\& n r m=i s o>$. Acesso em: 25 maio, 2010. doi: 10.1590/S0103-90161999000300023.

CARVAlHO, E.D. Linhagens triplóides de Pacu Piaractus mesopotamicus (Holmberg, 1887) (= Colossoma mitrei, Berg, 1895 e de Tambaqui Colossoma macropomum (Curvier, 1818): Indução artificial e estudos de sobrevivência e desempenho em condições de tanques de cultivo. 1992. 212f. Tese (Doutorado em Ecologia e Recursos Naturais) - Universidade Federal de São Carlos, SP.

CARVALHO-FILHO, J. As estatísticas da aqüicultura. Panorama da Aqüicultura, Botafogo, v.18, n.105, p.5759, 2008.

CAVERO, B.A.S. et al. Efeito da densidade de estocagem na homogeneidade do crescimento de juvenis de pirarucu em ambiente confinado. Pesquisa Agropecuária Brasileira, Brasília, v.38, n.1, p.103-107, 2003. 
CONTE, L. Produtividade e economicidade de tilapicultura em gaiolas na Região Sudoeste do Estado de São Paulo: estudos de casos. 2002. 73f. Dissertação (Mestrado em Agronomia) - Escola Superior de Agronômia “Luiz de Queiroz”, Universidade de São Paulo, SP.

FAO. State of World Aquaculture: 2006. Rome, 2006. 145p. (Fisheries Technical Paper 500).

FREITAS, M.J.C.C.; ANNUNCIATO, D.P. Botucatu às margens do Tietê cultura e sustentabilidade. Botucatu: Ministério da Cultura e AES Tietê, 2008. 158p.

GYLLENHAMMAR, A. et al. A mesocosm fish farming experiment and its implications for reducing nutrient load on a regional scale. Aquacultural Engineering, Seattle, v.38, p.117-126, 2008.

KUBITZA, F. “O mar está prá peixe... prá peixe cultivado”. Panorama da Aqüicultura, Botafogo, v.17, n.100, p.1423, 2007.

MARENGONI, N.G. Produção de tilápia do nilo Oreochromis niloticus (Linhagem Chitralada), cultivada em tanque-rede, sob diferentes densidades de estocagem. Archivos de Zootecnia, Córdoba, v.55, n.210, p.127-138, 2006.

MARTIN, N.B. et al. Custos e retornos na piscicultura em São Paulo. Informações Econômicas, São Paulo, v.25, n.1, p.9-47, 1995.

MEURER, F. et al. Fibra bruta para alevinos de tilápia do Nilo (Oreochromis niloticus, L.). Revista da Sociedade Brasileira de Zootecnia, Viçosa, v.32, n.2, p.256-261, 2003.

MORAES, A.M. et al. Avaliação econômica e zootécnica do cultivo de tilápia do Nilo Oreochromis niloticus em tanques-rede utilizando-se diferentes rações comerciais. In: AQUACIÊnCIA 2006, Bento Gonçalves, RS. Anais... Bento Gonçalves: Aquaciência, 2006. CDRoom. Para uso em PC

RIBEIRO, S. Governo quer ampliar receita da pesca e fortalecer indústria naval. 2007. Acesso em: 05 jan. 2009. Online. Disponível em: <http://www.ambienteemfoco.com.br/ ?cat $=27$ \&paged $=2>$.

SAMPAIO, J.M.C.; BRAGA, L.G.T. Cultivo de tilápia em tanques-rede na barragem do Ribeirão de Saloméa - Floresta Azul - Bahia. Revista Brasileira de Saúde e Produção Animal, Salvador, v.6, n.2, p.42-52, 2005.

STARLING, F.L.R.M.; LAZZARO, X.T. Controle da eutrofização em lagos e reservatórios tropicais por biomanipulação - Estudo de caso: Lago Paranoá (Brasília, Brasil). In: DE BERNARDI, R.; GIUSSANI, G. Biomanipulação para o gerenciamento de lagos e reservatórios - Diretrizes para o gerenciamento de lagos. São Carlos: ILEC/IIE, 2001. Cap.14, p.203221 .

VALENTI, W.C. Introdução. In: VALENTI, W.C. et al. Aqüicultura no Brasil: bases para um desenvolvimento sustentável. Brasília: CNPq Ministério da Ciência e Tecnologia, 2000. p.25-32.

VOLPATO, G. L.; FERNANDES, M. O. Social control of growth in fish. Brazilian Journal of Medical and Biological Research, Ribeirão Preto, v.27, p.797-810, 1994.

YI, Y. et al. Influence of Nile tilapia (Oreochromis niloticus) stocking density in cages on their growth and yield in cage and in ponds containing the cages. Aquaculture, Arden, v.146, p.205-215, 1996 . 